ISSN 2519-0652 CASUS.2017;2(3):190-199

\title{
Papel del enfermero en la nutrición de un recién prematuro con síndrome de Down
}

\section{Katherine Ortiz-Romanit Ruth Mucha-Montoya ${ }^{2}$}

RESUMEN: El síndrome de Down es una de las principales causas de mortalidad perinatal en prematuros con bajo peso al nacer. El presente reporte de caso presenta a una recién nacida pretérmino, abandonada por su madre, que se encontraba hospitalizada en un servicio de neonatología de un hospital público de Lima Norte por presentar trisomía 21, bajo peso, descarte de sífilis y riesgo de hipoglucemia. Ante esta situación se propuso ejecutar las intervenciones de enfermería priorizando la mejora de la alimentación y/o nutrición. Para ello, se seleccionaron y priorizaron una serie de diagnósticos de enfermería a través de los aspectos negativos de la valoración. El plan de cuidados se ejecutó en tres meses hasta su alta utilizando los 13 dominios de la taxonomía II NANDA-I, clasificación de los diagnósticos, intervenciones y resultados de enfermería. El papel del enfermero fue seleccionar adecuadamente una serie intervenciones que mejoraron los diagnósticos de enfermería: Interrupción de la lactancia materna, Desequilibrio nutricional inferior a las necesidades corporales, Riesgo de glucosa inestable y Riesgo de retraso en el desarrollo. Se recomienda que el profesional de enfermería ejecute intervenciones que favorezcan una buena alimentación al prematuro con discapacidad para prevenir el bajo peso u otra alteración nutricional o metabólica. Asimismo, capacitarse en la atención individualizada a $\mathrm{RN}$ con discapacidad.

PALABRAS CLAVE: Síndrome de Down; Recién nacido prematuro; Métodos de alimentación; Peso al nacer.

Citar como: Ortiz K, Mucha R. Papel del enfermero en la nutrición de un recién nacido prematuro con Síndrome de Down. CASUS. 2017;2(3):190-199.

1Universidad Católica Sedes Sapientiae. Licenciada en Enfermería. ${ }^{2}$ Hospital Nacional Sergio E. Bernales. Magíster en Docencia Universitaria. 


\section{INTRODUCCIÓN}

El síndrome de Down (SD) conocido también como trisomía del par 21 es considerada una discapacidad intelectual ocasionada por una alteración genética. La mayoría de los casos de SD se asocian a la edad materna avanzada (1). Por otro lado, existe un mayor riesgo de mortalidad perinatal en niños con SD con prematurez y bajo peso al nacer (2).

Se estima que cada año 276.000 recién nacidos (RN) mueren por un trastorno congénito. Entre estos trastornos el más frecuente es el síndrome de Down (1). Se calcula que 1 de cada 1100 recién nacidos presenta SD a nivel mundial (3). A nivel nacional el Consejo Nacional para la integración de las personas con discapacidad (CONADIS) informó que en 2015 se registraron 8800 personas con SD que equivale al $6.21 \%$ del total de registrados con discapacidad (4).

Un recién nacido con SD se caracteriza generalmente por hipotonía, boca pequeña, lengua protruyente, perfil facial plano, manchas de Bruschfield, etc. Cabe resaltar que solo en prematuros es complicado identificar si presenta SD, según estas características, en comparación a un RN a término (5). Además, padece una serie de alteraciones como estreñimiento, bajo peso, hipoglucemia, anomalías cardiacas, etc. (5). Mucho de ellos presentan dificultad en ganar peso en los primeros meses de vida, debido a trastornos de la succión o deglución, indicaciones nutricionales inadecuadas y cardiopatías congénitas (6). Se considera que no existe tratamiento para el SD, pero sí una serie de medidas de rehabilitación y cuidados especiales por parte de un equipo de profesionales de salud. Estas medidas y cuidados están enfocados a insertarlos a la sociedad para que actúen de manera autónoma y disfruten de una vida plena y digna (1).

A partir de este contexto el personal de enfermería, como parte del equipo de profesionales de salud, se enfrenta a un número creciente de RN pretérmino con alteraciones congénitas. Esta situación lleva consigo una serie de complicaciones como un incremento de la morbilidad, mayor tiempo de estancia hospitalaria y letalidad si no se implementan estrategias para poder actuar de una manera correcta y eficaz en el cuidado de enfermería al RN prematuro con alguna alteración congénita o discapacidad (7). Por esta razón, el objetivo del estudio fue ejecutar las intervenciones a un RN con síndrome de Down priorizando la mejora de la nutrición. Para ello, se aplicó el modelo de los
13 dominios de la taxonomía II de Nursing Diagnosis Classification (NANDA)-I (8) de Marjorie Gordon, considerada como una guía donde se pueden cumplir los objetivos o resultados según Nursing Outcomes Classification (NOC) (9) y desarrollar una serie de intervenciones de enfermería según Nursing Interventions Classification (NIC) (10). Estas taxonomías son empleadas para el desarrollo del cuidado enfermero como herramienta fundamental en el lenguaje de enfermería (8).

\section{PRESENTACIÓN DEL CASO}

Se presenta a una recién nacida pretérmino con bajo peso al nacer que nació por parto distócico (cesárea) con edad gestacional de 35 semanas (según método Capurro). Hija de madre multigesta con epilepsia y sífilis en tratamiento. Al Test de Apgar presentó 8 al minuto y 9 a los 5 minutos. La RN pasó al área de prematuros con un peso de $2200 \mathrm{~kg}$ y una glucosa 41 $\mathrm{mg} / \mathrm{dl}$. Se le diagnosticó trisomía 21 después de varios descartes, hipoglucemia y descarte de sífilis.

A los seis días de nacida el médico de guardia indica que pase a intermedios I (área de observación) por los antecedentes que presenta, así como los de su madre. En esta área recibió fórmula maternizada $30 \mathrm{ml}$ cada tres horas. Debido a la insistencia de la madre que podía dar de lactar se le entrega en sus brazos al bebé. En ese momento la madre manifiesta una crisis convulsiva donde la RN presenta una caída. Mediante una evaluación médica se diagnosticó equimosis en zona frontal izquierda, por lo que le indican que la $\mathrm{RN}$ pase a Intermedios II (área de infectados) y se le tome un examen de tomografía cerebral. Posteriormente, se le diagnosticó hematoma fronto temporal izquierda y traumatismo encéfalo craneano (TEC) leve sin signos de fractura. RN se quedó en Intermedios II.

\section{VALORACIÓN}

A los ocho días de nacida la bebe fue abandonada por su madre ocasionando una interrupción total de la lactancia materna exclusiva. Al momento la $\mathrm{RN}$ pesaba $2145 \mathrm{~kg}$. De acuerdo al análisis de glucosa presentó $91 \mathrm{mg} / \mathrm{dl}$ y respecto a la prueba de sífilis se descarta la patología y se suspende penicilina $G$ sódica. Los cuidados quedan a cargo de las enfermeras de turno enfocándose en los aspectos negativos de la valoración a nivel nutricional (ver tabla 1). 
Tabla 1. Análisis de los dominios según aspectos positivos y negativos

\begin{tabular}{|c|c|c|}
\hline Dominios & Positivos & Negativos \\
\hline Promoción de la Salud & No se observa. & $\begin{array}{l}\text { Síndrome de Down. } \\
\text { RN prematuro. }\end{array}$ \\
\hline Nutrición & $\begin{array}{l}\text { Recibe leche maternizada con } \\
\text { buena tolerancia oral } \\
\text { De acuerdo al control de glucosa } \\
\text { cada } 6 \text { horas RN presenta } 90 \\
\text { mg/dl (8 días de nacido). } \\
\text { Hemoglobina } 20.0 \mathrm{~g} / \mathrm{dL} \text { y } \\
\text { hematocrito } 60.9 \% \text {. }\end{array}$ & $\begin{array}{l}\text { Peso: } 2145 \mathrm{~kg} \\
\text { No recibe lactancia materna } \\
\text { exclusiva (abandono de la madre) } \\
\text { Glucosa } 41 \mathrm{mg} / \mathrm{dl} \text { (primer día de } \\
\text { nacido). }\end{array}$ \\
\hline Eliminación e Intercambio & $\begin{array}{l}\text { Micción espontánea. } \\
\text { Presencia de meconio. }\end{array}$ & No se observa. \\
\hline Actividad / Reposo & $\begin{array}{l}\text { Actividad moderada. } \\
\text { Presenta } 133 \text { pulsaciones por } \\
\text { minuto. }\end{array}$ & No se observa. \\
\hline Percepción y cognición & Llanto vigoroso. & No se observa. \\
\hline Autopercepción & No se observa. & No se observa. \\
\hline Rol / relaciones & No se observa. & $\begin{array}{l}\text { Madre abandona a RN. } \\
\text { Interrupción de la lactancia } \\
\text { materna. }\end{array}$ \\
\hline Sexualidad & $\begin{array}{l}\text { Genitales de acuerdo a su edad y } \\
\text { sexo. }\end{array}$ & No se observa. \\
\hline $\begin{array}{l}\text { Afrontamiento y tolerancia al } \\
\text { estrés }\end{array}$ & No se observa & No se observa. \\
\hline Principios vitales & No se observa. & No se observa. \\
\hline Seguridad/Protección & $\begin{array}{l}\text { Se encontró en cuna con una } \\
\text { temperatura corporal: } 36.5^{\circ} \mathrm{C} \text {. } \\
\text { Se descartó Sífilis y se suspendió } \\
\text { penicilina G. Sódica. }\end{array}$ & No se observa. \\
\hline Confort & RNPT se encontró en una cuna. & No se observa. \\
\hline Crecimiento/Desarrollo & No se observa. & $\begin{array}{l}\text { RNPT de } 35 \text { semanas de } \\
\text { gestación por Capurro. } \\
\text { Presentó bajo peso al nacer: } 2 \\
200 \mathrm{~kg} \text {. } \\
\text { Peso actual: } 2145 \text {. }\end{array}$ \\
\hline
\end{tabular}




\section{FASE DIAGNÓSTICA}

Reales:

- (00105) Interrupción de la lactancia materna r/c separación de la madre-niño e/p alimentación con vaso y cucharita. Dominio 2, Clase 1.

- (00002) Desequilibrio nutricional inferior a las necesidades corporales enfermedad actual e/p bajo peso: 2 145kg. Dominio, Clase 1.

\section{Potenciales:}

- (00179) Riesgo de glucosa inestable r/c con el retraso en el desarrollo. Dominio 2, Clase 4.

- (00112) Riesgo de retraso en el desarrollo r/c efectos de la discapacidad. Dominio 13, Clase 2.

\section{FASE DE PLANIFICACIÓN}

El plan de cuidados de enfermería se inició con la identificación de los aspectos negativos de la valoración priorizando una serie de diagnósticos de enfermería. A su vez, se seleccionaron las intervenciones para mejorar los objetivos y obtener buenos resultados de enfermería (ver tabla 2).

\section{FASE DE EJECUCIÓN}

Se ejecutaron las intervenciones y actividades de enfermería en tres meses en diferentes turnos de 12 horas diarios (ver tabla 2).

\section{FASE DE EVALUACIÓN}

En la tabla 2 se evidencia que la RN tuvo como diagnóstico de enfermería interrupción de la lactancia materna debido al abandono de la madre. Por ello, se tuvieron que ejecutar las intervenciones y actividades como la succión no nutritiva mediante el uso de chupetes limpios y la alimentación con vaso para una nutrición necesaria. El vaso debe ser peqseño 2(3y, 26\%łocar solo la mitad de leche maternizada inclinándolo hacia los labios del bebé mediante la asistencia del personal de enfermería, según la técnica correcta para lactante prematuro. La administración nutritiva fue realizada con afecto, calidad humana, paciencia (tiempo en las tomas debido a la lengua en protrusión de la $\mathrm{RN}$ ), trato delicado, cariñoso y sensible. Cabe resaltar que a la RN se le ejercitó para una buena succión, en espera de su madre y se le dio la leche maternizada mediante el vaso con el fin de evitar biberones y sonda orogástrica. Una vez aplicadas las intervenciones aumentaron los indicadores del NOC de un Likert 3 (A veces demostrado) a un Likert 5 (Siempre demostrado).
En cuanto al Desequilibrio nutricional inferior a las necesidades. Teniendo referencia el peso actual del RN $(2.145 \mathrm{~kg})$ se le proporcionaron cuidados del lactante prematuro y manejo de electrolitos, según la edad y el diagnóstico médico identificado. Estos son cuidados necesarios en la atención a su alimentación, estado de hidratación y eliminación. Se contabilizaron los ingresos y egresos de la ingesta que recibió cada 3 horas. La RN subió de peso a $2215 \mathrm{Kg}$ (Subió $70 \mathrm{~g}$ ) en menos de una semana. Se obtuvo como indicador Likert 3 (a veces demostrado) a un Likert 4 (frecuentemente demostrado).

Para el diagnostico Riesgo de glucosa inestable se trabajó mediante las intervenciones de enfermería manejo de hipoglicemia y monitorización nutricional con el fin de identificar signos y síntomas de hipoglucemia (tremores, diaforesis, somnolencia, rubor, hipotermia, etc.) previniendo una alteración neurológica o metabólica. Si en caso el enfermero identificaba hipoglicemia (glucosa en sangre $50 \mathrm{mg} / \mathrm{dl}$ ) se debía administrar glucosa según prescripción médica dextrosa al 10\% $2 \mathrm{ml} / \mathrm{Kg}$ vía endovenosa. Para ello se mantuvo una vía intravenosa (IV) permeable por prevención. Asimismo, se controló la glucosa en sangre según protocolo del establecimiento de salud. Se obtuvo como indicador Likert 3 (a veces demostrado) a un Likert 5 (siempre demostrado).

Por último, en el diagnóstico de enfermería de Riesgo de retraso en el desarrollo se aplicó la intervención Cuidado del lactante prematuro mediante la proporción un ambiente calmo en cada administración o toma de leche en un horario adecuado. Asimismo, se le brindó el afecto y simulación de calor materno por parte de la enfermera para mejorar la estimulación psicomotora y el estado neurológico, según su edad. Se obtuvo como indicador Likert 3 (a veces demostrado) a un Likert 5 (siempre demostrado).

\section{DISCUSIÓN}

En la evaluación del plan de cuidados se logró mejorar la nutrición del prematuro con síndrome de Down. Pese a la interrupción de la lactancia materna por parte de la madre se aplicó la succión no nutritiva mediante el uso de chupetes limpios (11). Esta técnica es recomendable para estimular al $\mathrm{RN}$ y acelerar la maduración de la succión. Como resultado facilita una transición rápida a la alimentación oral, además se evita la colocación de la sonda orogástrica que es un conocido factor estresante del RN (11). 
Tabla 2. Diagnósticos de enfermería: objetivos, intervenciones, actividades y resultados

\begin{tabular}{|c|c|c|c|c|}
\hline $\begin{array}{c}\text { Diagnósticos de } \\
\text { enfermería } \\
\text { NANDA I }\end{array}$ & $\begin{array}{c}\text { Objetivos } \\
\text { planteados NOC } 1\end{array}$ & $\begin{array}{c}\text { Intervenciones } \\
\text { de } \\
\text { enfermería NIC }\end{array}$ & Actividades de enfermería & Resultados NOC 2 \\
\hline $\begin{array}{l}\text { (00105) } \\
\text { Interrupción de la } \\
\text { lactancia materna } \\
\text { r/c separación de } \\
\text { la madre -niño e/p } \\
\text { alimentación con } \\
\text { vaso y cucharita } \\
\text { Dominio } 2 \text { : } \\
\text { Nutrición } \\
\text { Clases 1: Ingestión }\end{array}$ & $\begin{array}{l}1019 \text { Realización de alimentación } \\
\text { con vaso. } \\
\text { Prematuro lactante mejorará su } \\
\text { alimentación durante su } \\
\text { permanencia hospitalaria. } \\
\text { Indicadores: } \\
10904 \text { Utiliza la fórmula antes de la } \\
\text { fecha de caducidad } \\
101907 \text { Prueba la temperatura del } \\
\text { líquido antes de la toma } \\
101910 \text { Coloca la taza correctamente } \\
\text { durante la toma } \\
1019 \text { Realización de alimentación } \\
\text { con vaso } \\
\text { Likert } 3 \text { (a veces demostrado) } \\
0913 \text { Estado neurológico: función } \\
\text { sensitiva motora/ motora de pares } \\
\text { craneales. } \\
\text { Prematuro lactante mantendrá } \\
\text { reflejos de succión y deglución } \\
\text { durante su hospitalización. } \\
\text { Indicadores: } \\
091308 \text { Deglución } \\
091307 \text { Movimiento muscular facial } \\
091309 \text { Reflejo nauseoso } \\
\text { Likert } 3 \text { (a veces demostrado) }\end{array}$ & $\begin{array}{l} \\
6900 \text { Succión } \\
\text { no nutritiva }\end{array}$ & $\begin{array}{l}\text { Tener especial cuidado y afecto al } \\
\text { manipular a los pacientes con esta } \\
\text { patología. } \\
\text { Colocar en posición fowler, mientras } \\
\text { se sujeta su cabeza, cuello y espalda. } \\
\text { Mantener, apoyado sobre el labio } \\
\text { inferior del RN con los bordes de las } \\
\text { tasas en contacto con las partes } \\
\text { externas del labio superior. } \\
\text { Expulsar los gases del RN durante y } \\
\text { después de la toma. } \\
\text { Medir la ingesta y salida del RN en } 24 \\
\text { horas. } \\
\text { Seleccionar un chupete o sustituto de } \\
\text { chupete, con las normas establecidas } \\
\text { para evitar que obstruyan las vías } \\
\text { aéreas. } \\
\text { Colocar a la bebé de tal forma que la } \\
\text { lengua caiga sobre su base. } \\
\text { Colocar al bebé de tal forma el pulgar } \\
\text { o índice para favorecer el reflejo de } \\
\text { succión si fuera necesario. } \\
\text { Acariciar suavemente la mejilla del } \\
\text { bebé para estimular el reflejo de } \\
\text { succión. } \\
\text { Poner música suave y sin alterar el } \\
\text { desarrollo neurológico del prematuro. }\end{array}$ & $\begin{array}{l}\text { Prematuro recibe su } \\
\text { alimentación durante su } \\
\text { permanencia hospitalaria. } \\
\text { Indicadores: } \\
10904 \text { Utiliza la fórmula antes } \\
\text { de la fecha de caducidad } \\
101907 \text { Prueba la temperatura } \\
\text { del líquido antes de la toma } \\
101910 \text { Coloca la taza } \\
\text { correctamente durante la toma } \\
\text { 1019 Realización de } \\
\text { alimentación con vaso } \\
\text { Likert } 5 \text { (siempre demostrado) } \\
\text { RN mantiene sus reflejos de } \\
\text { succión y deglución durante su } \\
\text { hospitalización. } \\
\text { Indicadores: } \\
\text { 091308 Deglución } \\
\text { 091307 Movimiento muscular } \\
\text { facial } \\
\text { 091309 Reflejo nauseoso } \\
\text { Likert } 4 \text { (frecuentemente } \\
\text { demostrado). }\end{array}$ \\
\hline
\end{tabular}




\section{Tabla 2. Continúa}

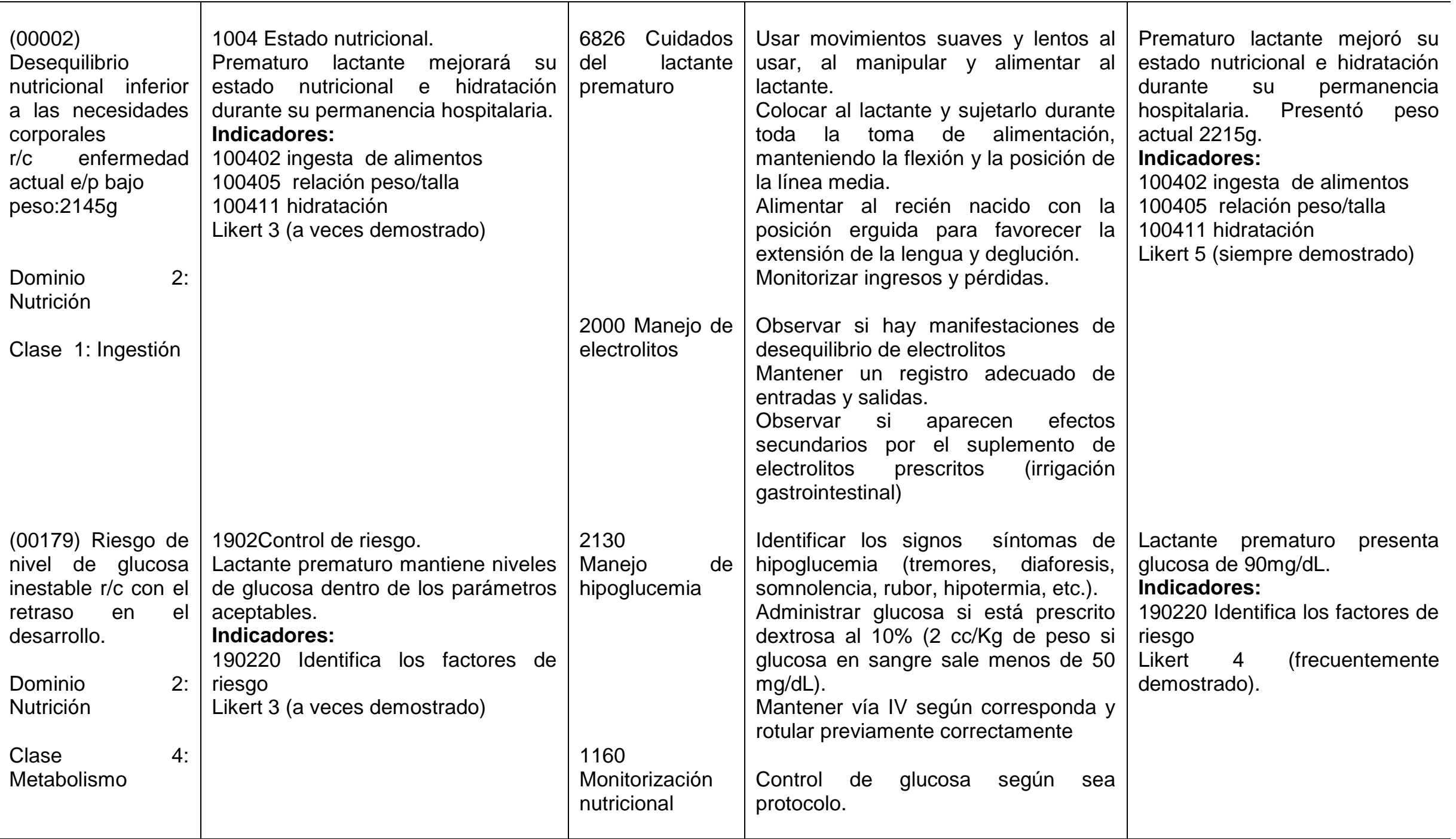


Tabla 2. Continúa

\begin{tabular}{|c|c|c|c|c|}
\hline & & & $\begin{array}{l}\text { Pesar al paciente diariamente y } \\
\text { registrarlo correctamente. } \\
\text { Vigilar las pérdidas y ganancias de } \\
\text { peso. } \\
\text { ldentificar cambios recientes en la piel } \\
\text { y estado nutricional del lactante } \\
\text { prematuro. } \\
\text { Evaluar la turgencia de la piel y } \\
\text { movilidad cutánea. } \\
\text { Observar la presencia de arcadas y } \\
\text { posible vómitos. } \\
\text { Identificar anomalías de la defecación, } \\
\text { como diarrea, sangre con moco y } \\
\text { defecación irregular. } \\
\text { Monitorizar la presencia de tejido } \\
\text { conjuntival: pálido, enrojecido y seco. } \\
\text { Evaluar la deglución (función motora } \\
\text { de los músculos faciales, orales y } \\
\text { linguales y reflejo nauseoso). }\end{array}$ & \\
\hline $\begin{array}{l}\text { (00112) } \\
\text { Riesgo de retraso } \\
\text { en el desarrollo r/c } \\
\text { efectos de la } \\
\text { discapacidad. } \\
\text { Dominio } 13 \\
\text { Crecimiento/Desarr } \\
\text { ollo } \\
\text { Clase } 2 \text { Desarrollo }\end{array}$ & $\begin{array}{l}0117 \text { Adaptación del prematuro. } \\
\text { Prematuro lactante mejorará su } \\
\text { desarrollo psicomotor según la edad } \\
\text { que le corresponde durante su } \\
\text { estancia hospitalaria. } \\
\text { Indicadores: } \\
011708 \text { tono muscular relajado } \\
011712 \text { sueño profundo } \\
01713 \text { sueño ligero } \\
01714 \text { despierto tranquilo } \\
01715 \text { despierto activo I } \\
01716 \text { atento a estímulos } \\
01717 \text { responde a estímulos } \\
01720 \text { interacción con el cuidador } \\
\text { Likert } 3 \text { (a veces demostrado) }\end{array}$ & $\begin{array}{l}6826 \text { Cuidados } \\
\text { del lactante } \\
\text { prematuro }\end{array}$ & $\begin{array}{l}\text { Monitorizar los estímulos en el entorno } \\
\text { del lactante y reeducarlo cuando sea } \\
\text { posible. } \\
\text { Modificar la } \\
\text { iluminación para } \\
\text { proporcionar un ritmo circadiano. } \\
\text { Programar los cuidados del lactante y } \\
\text { su alimentación según el ciclo de } \\
\text { sueño-vigilia. } \\
\text { Colocar al lactante y sujetarlo durante } \\
\text { la toma de alimentación, manteniendo } \\
\text { la flexión y la postura. } \\
\text { Monitorizar los ingresos y salidas. } \\
\text { Fomentar la lactancia materna. }\end{array}$ & $\begin{array}{l}\text { Prematuro lactante mantiene el } \\
\text { desarrollo psicomotor cerca a la } \\
\text { edad que le corresponde } \\
\text { durante su estancia hospitalaria. } \\
\text { Indicadores: } \\
011708 \text { tono muscular relajado } \\
011712 \text { sueño profundo } \\
01713 \text { sueño ligero } \\
01714 \text { despierto tranquilo } \\
01715 \text { despierto activo } \\
01716 \text { atento a estímulos } \\
01717 \text { responde a estímulos } \\
01720 \text { interacción con el } \\
\text { cuidador } \\
\text { Likert } 5 \text { (siempre demostrado) }\end{array}$ \\
\hline
\end{tabular}


Asimismo, se dio la alimentación de leche maternizada en un vaso (12). Este procedimiento no interfiere la succión y no crea confusión de pezón como en el caso de biberones (13). Cabe resaltar que el uso del vaso es una manera eficaz de alimentación no solo a los RN sanos y a término, sino también en pacientes prematuros con SD como se evidenció en el estudio.

Respecto al diagnóstico Desequilibrio nutricional inferior a las necesidades corporales que tenía el RN con SD se mejoró gracias a la aplicación de cuidados y manejo de electrolitos (5). En caso de abandono de un RN hospitalizado la enfermera es responsable de cuidado de la alimentación del mismo (toma el rol de la madre) dando leche maternizada en una posición correcta del $\mathrm{RN}$, monitorizando los ingresos (cantidad adecuada de leche maternizada, electrolitos, etc.) y pérdidas o eliminación, peso diario del paciente, etc. Estas acciones se realizan con el fin de que el paciente no baje de peso, sino que aumente adecuadamente durante la estancia hospitalaria (7).

Por otro lado, en el diagnóstico Riesgo de glucosa inestable se logró mantener una glucosa en sangre dentro de los valores normales $(91 \mathrm{mg} / \mathrm{dl})$ mediante las intervenciones de enfermería manejo de hipoglicemia y monitorización nutricional (5). En pacientes con SD es importante un seguimiento de la glucosa cada 24 horas por la inestabilidad del parámetro que presentan estos pacientes. En caso que no esté dentro de sus valores normales se mide la glucosa cada 6 horas al día. Es importante identificar una hipoglucemia mediante el control de glucosa y la identificación de signos que presenta un $\mathrm{RN}$ con SD para poder administrar dextrosa vía endovenosa según el peso del paciente con el fin de impedir trastornos tipo neurológicos y endocrinológicos a futuro (14).

Por último, el diagnóstico Riesgo de retraso en el desarrollo se previno a través de los cuidados individualizados al lactante prematuro (15). Esta intervención está enfocada a reducir los factores estresantes del ambiente, programar el horario de alimentación para no interrumpir el sueño del paciente, verificar su estado de alerta, organización sueño-vigilia con la finalidad de estructurar, facilitar y mantener el estado de conciencia del RN evitando extenuación y agitación del mismo gracias a un ambiente calmo y agradable. Asimismo, favorece la ganancia de peso (15). Por otro lado, al estar despierto estará más conectado para una interacción positiva y adecuada con las personas que están a su alrededor.

Entre las dificultades del estudio se encontró que, según la situación problemática del RN, se necesitaba de un banco de leche materna dentro del establecimiento de salud y no alimentar solo con leche maternizada (fórmulas). Cabe resaltar que en este estudio se pudo implementar una práctica individualizada a la paciente mediante intervenciones en un tiempo adecuado (tres meses).

Se recomienda que el profesional de enfermería ejecute intervenciones que favorezcan una buena alimentación al RN prematuro con SD para prevenir y tratar el bajo peso u otra alteración nutricional o metabólica. Asimismo, se debe capacitar en la atención individualizada al RN con alguna discapacidad. Igualmente se deben crear guías o protocolos estandarizados finalizados al cuidado del RN con discapacidad. Por otro lado, los profesionales de salud deben apoyar a las madres con un bebé con SD u otra discapacidad con el fin de prevenir situaciones de abandono o rechazo de su propio hijo.

\section{REFERENCIAS BIBLIOGRÁFICAS}

1. Organización Mundial de la Salud (OMS). Anomalías congénitas [internet]. Ginebra, Suiza: OMS; 2017 [citado el 20 de setiembre de 2017]. Disponible en: http://www.who.int/mediacentre/factsheets/fs37 0/es/

2. Sparks T N, Griffin E, Page J, Pilliod R, Shaffer $\mathrm{BL}$, Caughey AB. Down syndrome: perinatal mortality risks with each additional week of expectant management. Prenatal diagnosis. 2016; 36(4):368-374.

3. Naciones unidad. Día mundial del síndrome de Down, 21 de marzo [internet]. Nueva York, Estados Unidos: 2017 [citado el 20 de setiembre de 2017]. Disponible en: http://www.un.org/es/events/downsyndromeday /background.shtml 
4. Consejo Nacional para la integración de las personas con discapacidad (CONADIS). Informe temático $\mathrm{n}^{\circ}$ 2: Síndrome de Down en el Perú [Internet]. Lima, Perú: 2015 [citado el 21 de setiembre de 2017]. Disponible en: http://www.conadisperu.gob.pe/observatori o/index.php/informacion-debase/informes/nacional/tematicos/325informe-tematico-n-2-sindrome-de-downen-el-peru

5. Lizama M, Retamales N, Mellado C. Recomendaciones de cuidados en salud de personas con síndrome de Down: 0 a 18 años. Revista médica de Chile. 2013;141(1):80-89.

6. Baum RA, Nash PL, Foster JEA, Spader M, Ratliff-Schaub K, Coury DL. Primary Care of children and adolescents with Down Syndrome: An Update. CurrProbl Pediatr Adolesc Health Care 2008; 38(8):241-61.

7. Mata M, Salazar ME, Herrera LR. Cuidado enfermero en el recién nacido prematuro. Rev Enferm Inst Mex Seguro Soc. 2009; 17(1):45-54.

8. Herdman TH, Kamitsuru S. NANDA internacional. Diagnósticos enfermeros: Definiciones y clasicación, 2015-2017. 10ma ed. Barcelona: Elsevier; 2015.

9. Moorhead S, Johnson M, Maas ML, Swanson E. Clasificación de Resultados de Enfermería (NOC). 5ta ed. Barcelona: Elsevier; 2014.

10. Bulechek GM, Butcher HK, Dochterman JM, Wagner CM.

Clasificación de Intervenciones de Enfermería (NIC). 6ta ed. Barcelona: Elsevier; 2014.

11. Martínez L, Díaz EP, García, S, Martí J. Uso del chupete: beneficios y riesgos. Anales españoles de pediatría. 2000;53(6):580-585.

12. Asociados C. Primer consenso nacional sobre alimentación en el primer año de la vida. Acta Pediátrica de México.2007;28(5):213-41.
13. Flint A, New K, Davies M. W. Cup feeding versus other forms of supplemental enteral feeding for newborn infants unable to fully breastfeed. Cochrane Database Syst Rev. 2008;2(2):1-23.

14. Caballero J. Efectos neurológicos de la hipoglucemia en el paciente diabético. Revista de Neurología. 2016; 63(6): 262-268.

15. Schapira, IT, Parareda V, Coria MB, Roy E. propuesta de intervención ambiental y en el desarrollo de recién nacidos de alto riesgo. Revisión bibliográfica. Rev. Hosp. Mat. Inf. Ramón Sardá. 1994;13(3):101-109. 
ISSN 2519-0652 CASUS.2017;2(3):190-199

\section{Role of the nurse in the nutrition of a newborn with Down Syndrome}

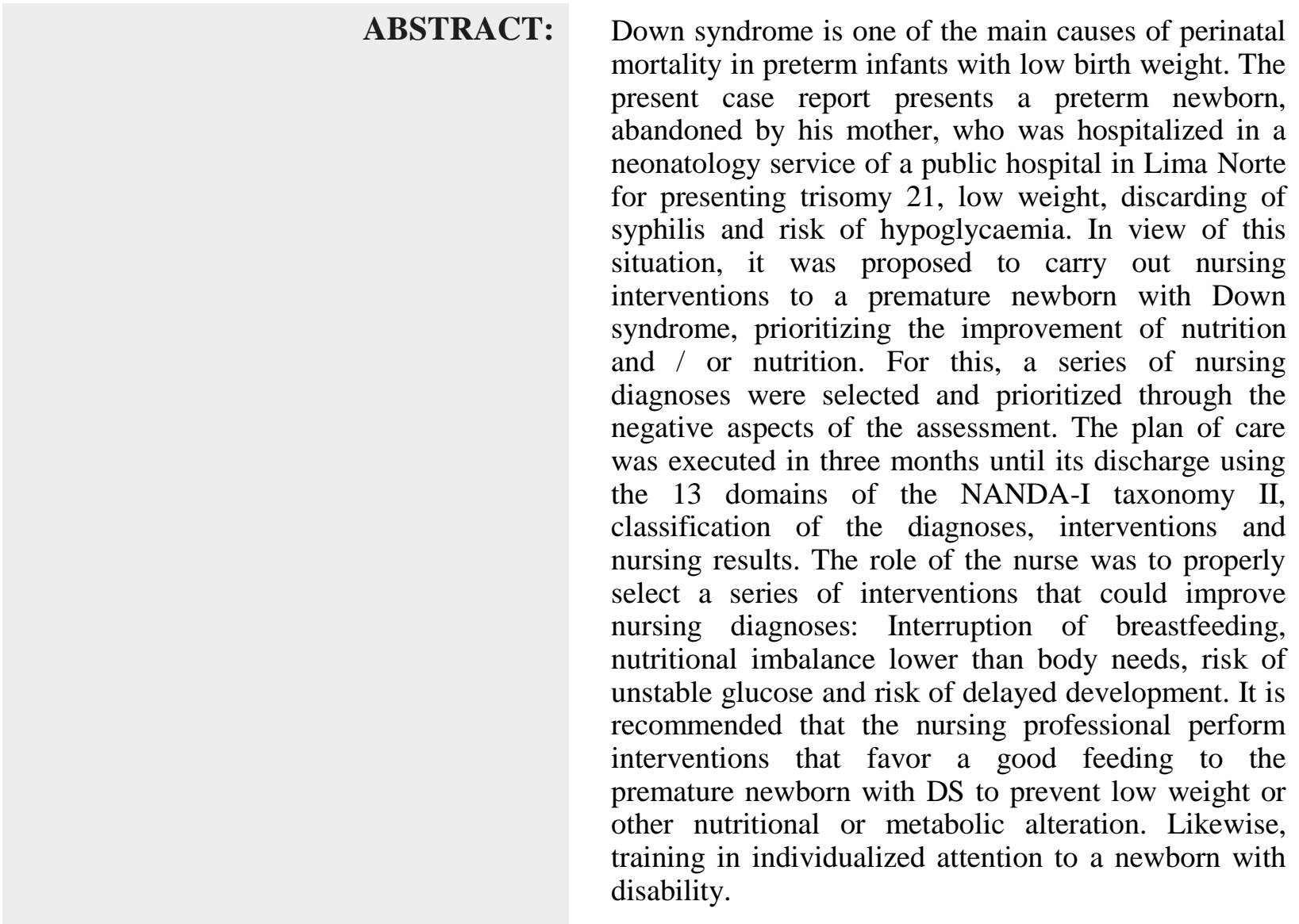

KEY WORDS: Down syndrome; Premature; Feeding Methods; Birth Weight. 